\title{
A Case of Susac Syndrome
}

\author{
Han Joo Cho, Chul Gu Kim, Sung Won Cho, Jong Woo Kim \\ Department of Ophthalmology, Kim's Eye Hospital, Konyang University College of Medicine, Seoul, Korea
}

\begin{abstract}
The purpose of this article is to report on the first known Korean case of Susac syndrome. An 18-yearold female came to our clinic reporting blurred vision of the left eye for 2 days. She also complained of decreased hearing with tinnitus of the right ear and mild headache. She was previously healthy and had no remarkable medical history. Best-corrected visual acuity was $20 / 50$ in the left eye and 20 / 20 in the right eye. An axiomatic triad of ocular, cochlear, and neurologic involvement was observed in the patient. Fluorescein angiography showed branched retinal arterial occlusions in the left eye. A sudden right sensorineural hearing loss was observed on audimetry. Magnetic resonance images showed a hyperintense lesion in the white matter around the corpus callosum. The patient was treated with high doses of systemic corticosteroids, and no neuropsychological sequelae were observed. This is the first case report of Susac syndrome in Korea. In cases of retinal arterial occlusion with hearing loss or neuropsychological symptoms, Susac syndrome should be suspected.
\end{abstract}

Key Words: Encephalopathy, Hearing loss, Retinal artery occlusion, Susac syndrome

Susac syndrome is a rare disease with a putative autoimmune vasculitic mechanism resulting in microangiopathy affecting the arterioles of the brain, retina and cochlea. It is characterized by a clinical triad of subacute encephalopathy, visual loss secondary to retinal branch occlusions and sensorineuronal hearing loss [1]. After the first report by Susac et al. [1], approximately 80 cases have been reported, with a predominance of young females [2,3]. Here, we present the first Korean case of Susac syndrome.

\section{Case Report}

An 18-year-old female came to our clinic reporting blurred vision of the left eye for 2 days. She also complained of de-

Received: August 26, 2011 Accepted: October 23, 2011

Corresponding Author: Han Joo Cho, MD. Kim's Eye Hospital, \#136, Yeongsin-ro, Yeongdeungpo-gu, Seoul 150-034, Korea. Tel: +82-2-26397777, Fax: +82-2-2639-7766, E-mail: ccnnrr@naver.com creased hearing with tinnitus of the right ear and mild headache. She was previously healthy and had no remarkable medical or ocular history. Best-corrected visual acuity (BCVA) was 20/50 in the left eye, and 20/20 in the right eye. The anterior segments of both eyes were unremarkable. Fundus examination and fluorescein angiography of the left eye showed ischemic retina with signs of branch retinal artery obstruction (Fig. 1).

We performed brain magnetic resonance imaging (MRI) and lumbar puncture to evaluate any brain or central nervous system lesions. The MRI scan showed a T2-weighted, high-signal, discrete area around the periventricular region, in the white matter and in the corpus callosum (Fig. 2). The lumbar puncture revealed a high protein level $(1.8 \mathrm{~g} / \mathrm{L})$, but no oligoclonal bands.

Blood pressure, echocardiography, carotid ultrasonography, full blood count, erythrocyte sedimentation rate, fasting lipids and glucose, autoantibody screen including anticardiolipin antibody, protein $\mathrm{S}$ and $\mathrm{C}$ levels, and antithrombin III level were all normal. An audiogram showed moderate 

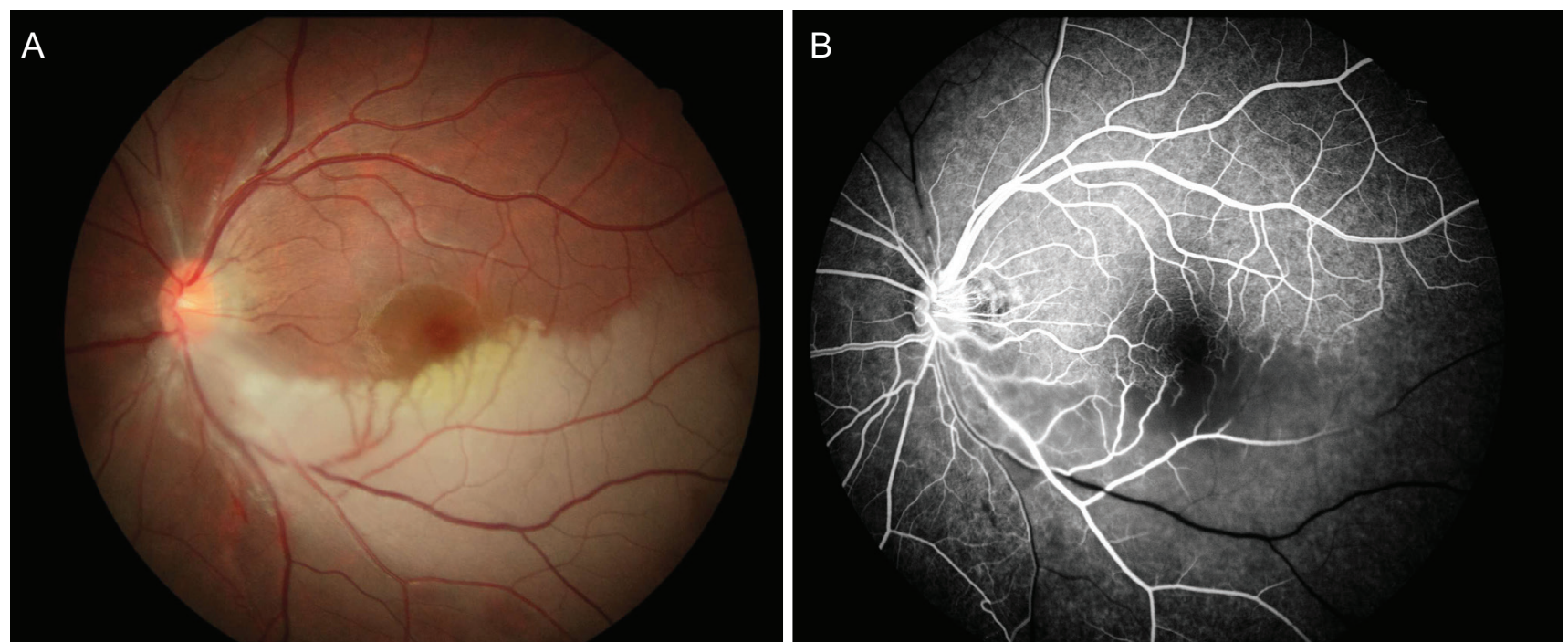

Fig. 1. (A) Fundus photography of the left eye showing ischemic retinal edema inferotemporally and narrowed branch artery superonasally. (B) Fluorescein angiography of the same patient showing arterial wall hyperfluorescence and branch retinal artery obstruction.
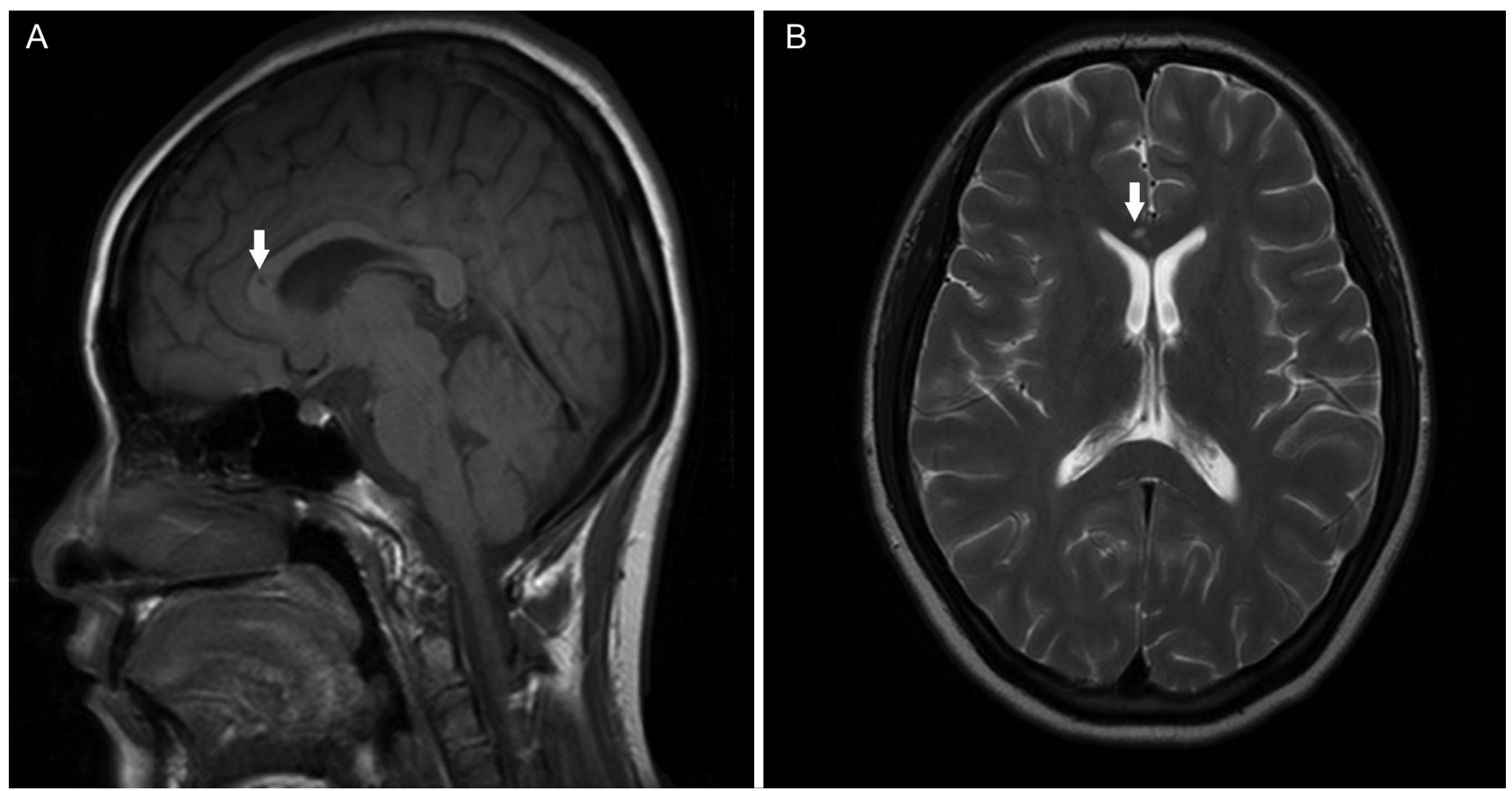

Fig. 2. (A) Mid sagittal T1-weighted magnetic resonance imaging (MRI) of the same patient showing punctuate hypointense foci within the corpus callosum (arrow). (B) Transverse, T2-weighted MRI showing a hyperintense foci in the mid white matter around the corpus callosum (arrow).

sensorineural hearing loss in the right ear.

The patient was treated with intravenous prednisolone 1 $\mathrm{g} / \mathrm{kg}$ for 3 days followed by oral steroid tapering. Three months after treatment, BCVA recovered to $20 / 25$ in the left eye. A mild residual auditory defect remained, but no remaining tinnitus or headache were observed.

\section{Discussion}

Susac syndrome was first described by Susac in 1976 and since then has been called by different names, including RED-M (retinopathy, encephalopathy, deafness associated with microangiopathy), SICRET (small infarction of 
cochlear, retina and encephalitic tissue), and retinocochlear vasculopathy $[1,2]$. This syndrome is rare and usually shows female predominance with a sex ratio of 3 to 1 . Approximately 80 cases of Susac syndrome have been reported in the literature [1-4].

The syndrome is often misdiagnosed and should be differentiated from a number of other diseases, including multiple sclerosis, disseminated encephalomyelitis, lupus erythematosus, Ménière disease, migraine and thromboembolic strokes [5]. Moreover, many patients do not present initially with the clinical triad and show incomplete forms of the disease in which only branch retinal artery obstruction or hearing loss in the absence of encephalopathy is observed [6].

Brain MRI, fluorescein angiography and audiometry are essential for correct diagnosis. MRI findings include multiple hyper-intense small lesions through infratentorial structures [5]. Susac and colleagues reported that there was always involvement of the corpus callosum, and callosal lesions typically involve the central fibers with relative sparing of the periphery [7]. Multiple sclerosis can be differentiated based on these findings. Fluorescein angiography showed characteristic retinopathy with multiple retinal artery occlusions. Yellow retinal arterial wall plaque in association with Susac syndrome has been described [8], but plaques were not identified in our patient. The bilateral or unilateral hearing loss is due to cochlear involvement. Hearing loss may be asymptomatic and found only by audimetry. This hearing loss typically involves low and medium frequencies [3-5].

The pathogenesis of this syndrome is believed to be an immunological reaction, leading to small vessel vasculitis causing micro infraction in the retina, brain and apical turn of the cochlea $[3,5]$. In a recent autopsy study, the findings of retinas with Susac syndrome suggested that the syndrome could be an endothelioapthy [9]. The disease course is known to be monophasic and self-limited, with a duration of 1 to 4 years $[3,6]$.

Different therapeutic protocols such as corticosteroids, immunosuppressive agents, anti-platelet agents, anticoagulant agents and plasmapheresis have been reported. Although their efficacy remains difficult to evaluate, early recognition of the disease is important because treatment with immunosuppression seems to reduce permanent cog- nitive, audiologic, and visual sequelae [3,5].

To the best of our knowledge, this is the first report of Susac syndrome in Korea. A high index of suspicion leading to early recognition of this syndrome is important because immunosuppressive treatment may decrease permanent neuropsychological or visual impairment. In cases with retinal arterial occlusion with hearing loss or neuropsychological symptom, early detection of the characteristic brain MRI finding, audimetry, and funduscopic examination should be considered.

\section{Conflict of Interest}

No potential conflict of interest relevant to this article was reported.

\section{References}

1. Susac JO, Hardman JM, Selhorst JB. Microangiopathy of the brain and retina. Neurology 1979;29:313-6.

2. Murata Y, Inada K, Negi A. Susac syndrome. Am J Ophthalmol 2000;129:682-4.

3. O'Halloran HS, Pearson PA, Lee WB, et al. Microangiopathy of the brain, retina, and cochlea (Susac syndrome): a report of five cases and a review of the literature. Ophthalmology 1998;105:1038-1044.

4. Do TH, Fisch C, Evoy F. Susac syndrome: report of four cases and review of the literature. AJNR Am J Neuroradiol 2004;25:382-8

5. Papo T, Biousse V, Lehoang P, et al. Susac syndrome. Medicine (Baltimore) 1998;77:3-11.

6. Saw VP, Canty PA, Green CM, et al. Susac syndrome: microangiopathy of the retina, cochlea and brain. Clin Experiment Ophthalmol 2000;28:373-81.

7. Susac JO, Murtagh FR, Egan RA, et al. MRI findings in Susac's syndrome. Neurology 2003;61:1783-7.

8. Egan RA, Ha Nguyen T, Gass JD, et al. Retinal arterial wall plaques in Susac syndrome. Am J Ophthalmol 2003;135:483-6.

9. McLeod DS, Ying HS, McLeod CA, et al. Retinal and optic nerve head pathology in Susac's syndrome. Ophthalmology 2011;118:548-52. 